\title{
Periocular basal cell carcinoma: recurrence risk factors/ when to reoperate?
}

\author{
Krzysztof Gąsiorowski¹, Katarzyna Iwulska², Jan Zapała', Grażyna Wyszyńska-Pawelec ${ }^{1}$
}

${ }^{1}$ Department of Cranio-Maxillofacial Surgery, Jagiellonian University Medical College, The University Hospital, Krakow, Poland 2Department of Maxillofacial Surgery, L. Rydygier Hospital, Krakow, Poland

Adv Dermatol Allergol 2020; XXXVII (6): 927-931

DOI: https://doi.org/10.5114/ada.2020.102109

\begin{abstract}
Introduction: The aim of periocular basal cell carcinoma (BCC) surgery is radical excision of the tumour while maintaining eyelid functionality and facial aesthetics. Differences in management of the patients after excision of eyelid BCC with a narrow margin are described in the literature.

Aim: This study concerns the correlation between the recurrence rate in the periorbital area and the narrow safety margin of excision in the histopathological report with respect to various clinical and histopathological features. Material and methods: A retrospective non-randomized analysis was conducted on 158 patients with BCC of the periorbital area. These patients were operated on between January 2002 and December 2016. A database was created, comprised of patient age, sex, location of the lesion, TNM, method of reconstruction, result of the histopathological examination, date and location of the recurrence.

Results: In 66 (41.77\%) patients BCC was radically removed. In 50 (31.65\%) patients BCC was removed with a narrow margin and in $42(26.58 \%)$ cases, radical excision was not achieved. The recurrence rate was significantly higher in the aggressive BCC group compared to those with non-aggressive BCC $(p=0.004)$. The recurrence-free rate for non-aggressive subtypes was $98.11 \%$ in both the first and fifth years, but in aggressive subtypes it was $89.06 \%$ in the first year but fell to $80.16 \%$ in the fifth year.

Conclusions: An aggressive subtype of BCC significantly influences the risk of non-radical excision of the lesion. Aggressive BCC subtypes should have more frequent check-ups. There is no need to reoperate patients with a narrow margin of BCC excision.
\end{abstract}

Key words: recurrence rate, periocular, incomplete excision, basal cell carcinoma, risk factors.

\section{Introduction}

Basal cell carcinoma (BCC) is the most common skin cancer occurring between the ages of fifty and seventy. About $80 \%$ of the changes are located in the head and neck area. Of these lesions, the BCC of the orbital region accounts for approximately 20\% [1] and in the eyelid, $10 \%$ [2]. The clinical symptom of BCC is a slowly growing nodule or ulcer usually located on the lower eyelid and in the medial canthal region of the eye, rarely metastasizing. Local recurrences vary from $0.39 \%$ to $4 \%$ and cause morbidity due to involvement of important functional structures (content of the orbit, eyeball, anterior skull base). Anatomic location in the $\mathrm{H}$-zone and periorbital area has been known to be the risk factor for BCC recurrence. Also aggressive BCC subtypes such as micronodu- lar, infiltrating, sclerosing, morpheaform tend to recur more often compared to the nodular subtype. According to the National Comprehensive Cancer Network (NCCN) 2019, the treatment of choice for high-risk BCC is Mohs micrographic surgery (MMS) or complete circumferential peripheral and deep margin assessment with permanent or intraoperative frozen section analysis [3].

\section{Aim}

This study concerns the correlation between the recurrence rate in the periorbital area and the narrow safety margin of excision in the histopathological report with respect to various clinical and histopathological features.

Address for correspondence: Grażyna Wyszyńska-Pawelec, Department of Cranio-Maxillofacial Surgery, Jagiellonian University Medical College, The University Hospital, 2 Macieja Jakubowskiego St, 30-688 Krakow, Poland, phone: +48 124002800 , fax: +48 124002800 , e-mail: grazyna.wyszynska-pawelec@uj.edu.pl Received: 31.01.2019, accepted: 28.04.2019. 


\section{Material and methods}

A retrospective analysis was performed on a group of 158 patients with BCC of the periorbital area. The patients were operated on between January 2002 and December 2016 in the Department of Maxillofacial Surgery of the Jagiellonian University in Krakow. Inclusion criteria were as follows: patients with primary BCC, and single neoplasm. Patients treated previously or at present for cancers in other locations as well as patients treated with immunosuppressants were not included into this study. The incisional and punch biopsies or recurrent lesions were excluded. The database included for each patient: age, sex, location of the lesion, TNM (NCCN 2019), method of reconstruction, the result of the histopathological examination, date and location of recurrence (if any), and date of the last follow-up visit.

All patients underwent standard surgical excision with a margin of $4 \mathrm{~mm}$ of surrounding healthy tissue as the safety margin (Figure 1). In some cases, an intraoperative histopathological examination was performed. Excised lesions were verified according to the 2006 WHO classification. All collected data were analysed according to the local recurrence rate. The specimens were divided into three groups on the basis of pathology results at the time of primary surgical removal: non-radical excision (positive, involved margin), excision with narrow margins $<2 \mathrm{~mm}$, and radical excision with margin $\geq 2 \mathrm{~mm}$. According to the NCCN 2019, the aggressive BCC included infiltrative, micronodular and morpheaform subtypes and the non-aggressive - nodular and superficial. Presence of vessels or nerves infiltration was not indicated in the histopathological report.

\section{Statistical analysis}

The Kaplan-Meier curves were used to determine the relapse-free survival rate. The comparison of KaplanMeier curves was made using the log-rank test. The significance level of 0.05 was assumed in the analysis. Odds ratio was used to determine the risk of recurrence depending on the BCC subtype. The analysis was carried out in the R program, version 3.4.3.

\section{Results}

In our study we included 158 patients with histopathologically confirmed primary BCC of the orbital region. There were 80 (50.63\%) males and 78 (49.37\%) females. The average age was $68 \pm 12.43$. The time between the first symptoms observed by patients and a performed biopsy in the outpatient clinic was 45 months, with a median time of 24 months (12-60). In 88 (55.7\%) patients, BCC developed in the medial canthal region. Table 1 shows the study group characteristics according to the recurrence.

The average follow-up time was 37 months with a median follow-up time of 22 months (minimum 1 month, maximum 130 months).

One hundred thirty patients were operated under general and 28 under local anaesthesia. As a rule, after excision of cancer, all surgical instruments as well as surgical gloves of the team were changed to prevent the transfer of tumour cells. In 66 (41.77\%) patients, BCC was radically removed. In 50 (31.65\%) patients BCC was removed with a narrow margin and in 42 (26.58\%) of cases, radical excision was not achieved. In the group
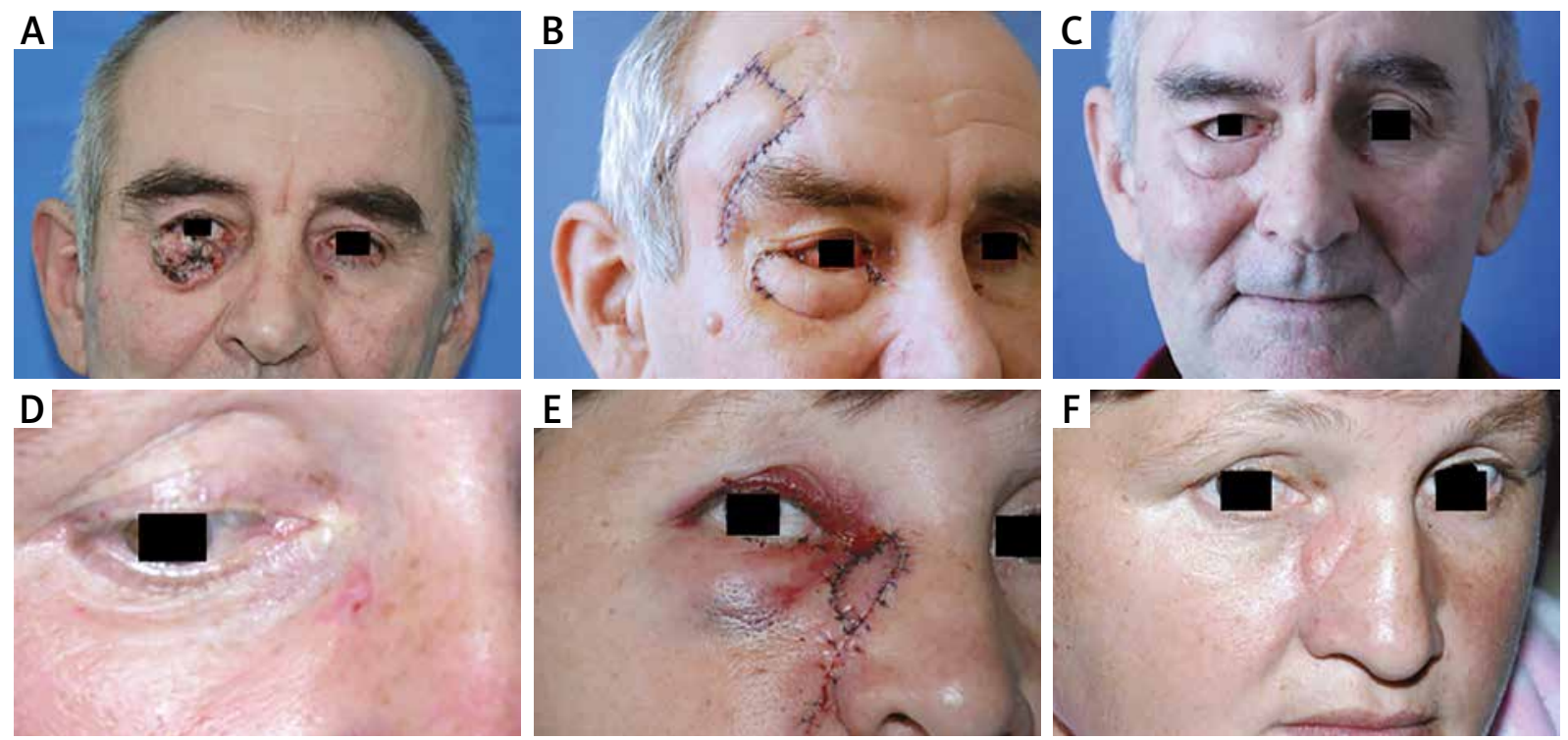

Figure 1. Early and late results of local flaps used for the reconstruction after excision of periocular BCC 
Table 1. Study group characteristics according to the recurrence

\begin{tabular}{|c|c|c|c|}
\hline Parameter & $\begin{array}{l}\text { BCCs with no } \\
\text { recurrence }\end{array}$ & $\begin{array}{l}\text { BCCs with } \\
\text { recurrence }\end{array}$ & $P$-value \\
\hline Age & $68(20-95)$ & $66(51-82)$ & \\
\hline Sex $M: F$ & $76: 76$ & $4: 2$ & \\
\hline \multicolumn{4}{|l|}{ Localization: } \\
\hline Medial canthal region & 85 & 3 & \multirow[t]{4}{*}{0.377} \\
\hline Upper eyelid & 9 & 1 & \\
\hline Lower eyelid & 50 & 1 & \\
\hline Lateral canthal region & 8 & 1 & \\
\hline \multicolumn{4}{|l|}{ TNM: } \\
\hline T1NOMO & 10 & 1 & \multirow[t]{4}{*}{0.229} \\
\hline T2NOMO & 91 & 3 & \\
\hline T3NOMO & 51 & 2 & \\
\hline T4NOMO & 0 & 0 & \\
\hline \multicolumn{4}{|l|}{ Excision: } \\
\hline Radical & 66 & 0 & \multirow[t]{3}{*}{0.002} \\
\hline Narrow margins & 50 & 0 & \\
\hline Non-radical & 36 & 6 & \\
\hline \multicolumn{4}{|l|}{ Histological type: } \\
\hline Aggressive & 15 & 5 & \multirow[t]{2}{*}{0.027} \\
\hline Non-aggressive & 137 & 1 & \\
\hline \multicolumn{4}{|l|}{ Margin involved: } \\
\hline Deep & 29 & 3 & \multirow[t]{3}{*}{0.729} \\
\hline Lateral & 32 & 1 & \\
\hline Both & 25 & 2 & \\
\hline
\end{tabular}

of incomplete excision of BCC, 13 patients were reoperated and in 9 patients excisional biopsy of the scar was performed during the second stage of treatment. In all cases BCC cells were not found in histopathological examination. Twenty patients refused to have proposed reoperation, but agreed to more frequent check-ups in our outpatient clinic.

Recurrence of BCC was observed in 6 (3.8\%) patients. The average time to recurrence was 26 months, and the median was 8 months (range: 2-113 months).

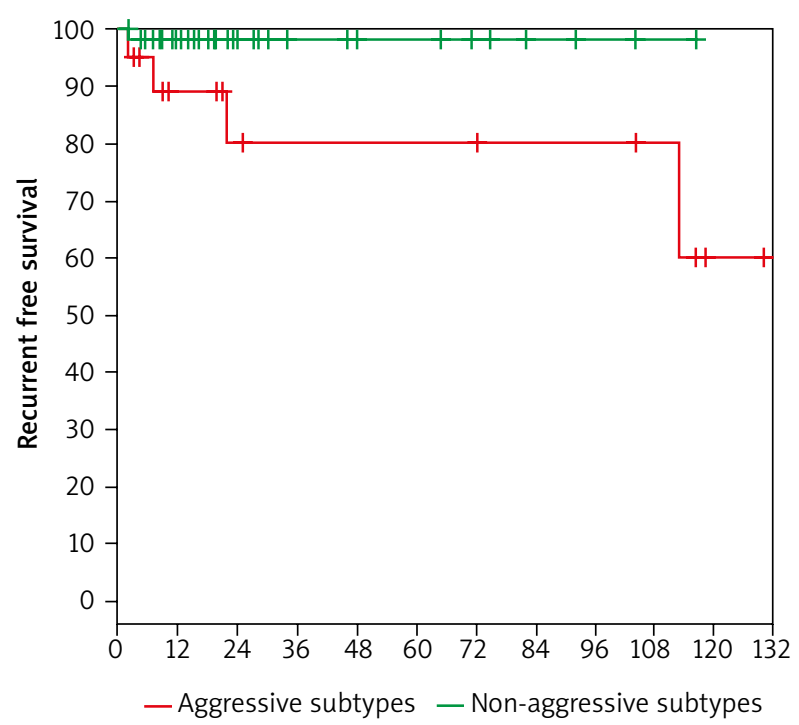

Figure 2. Analysis of risk factors for recurrence

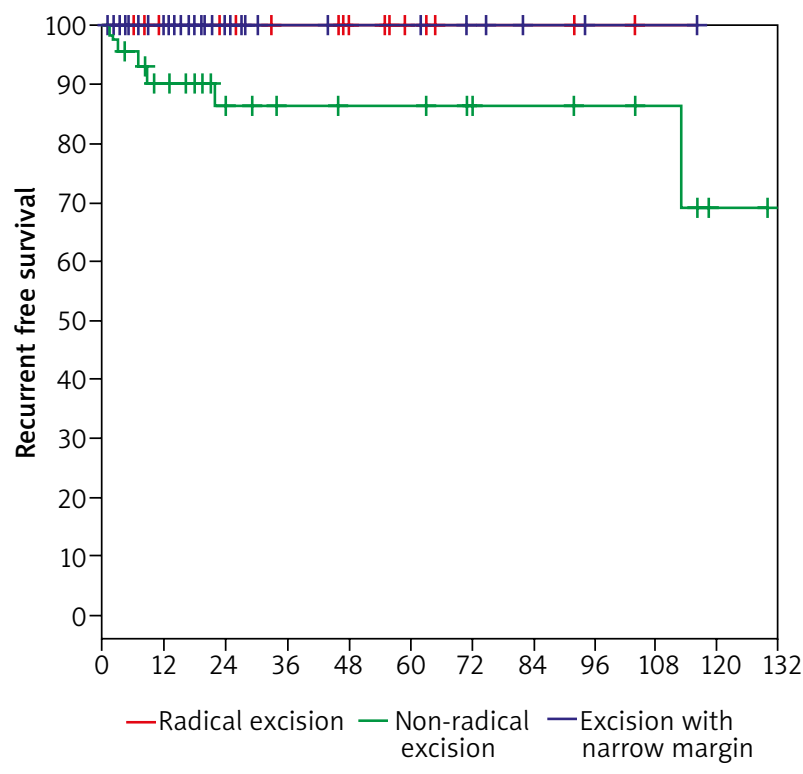

Figure 3. Analysis of risk factors for recurrence

Table 2. Analysis of risk factors for non-radical excision of BCC

\begin{tabular}{lcccccc}
\hline Subtypes & $\boldsymbol{N}$ & Non-radical excision & OR & \multicolumn{2}{c}{$95 \% \mathrm{Cl}$} & $P$-value \\
\hline Aggressive subtypes & 20 & 11 & 1 & Ref. & & 0.004 \\
\hline Nonaggressive subtypes & 138 & 31 & 0.2 & 0.067 & 0.601 & $P$-value \\
\hline Localization & $\boldsymbol{N}$ & Non-radical excision & OR & \multicolumn{2}{c}{$95 \% \mathrm{Cl}$} & \\
\hline Medial canthal region & 88 & 23 & 1 & Ref. & & 0.547 \\
\hline Lower eyelid & 51 & 11 & 0.777 & 0.343 & 1.763 & 0.358 \\
\hline Upper eyelid & 10 & 4 & 1.884 & 0.488 & 7.279 & 0.253 \\
\hline Lateral canthal region & 9 & 4 & 2.261 & 0.559 & 9.151 & \\
\hline
\end{tabular}


The recurrence rate was significantly higher in the group of aggressive BCC compared to non-aggressive $(p=0.004)$. Five out of six recurrent BCCs were aggressive subtypes, in 3 cases they were morphea type, and in 2 cases styloid. The recurrence-free rate for nonaggressive subtypes was $98.11 \%$ in both the first and fifth year, but in aggressive subtypes it was $89.06 \%$ in the first year but fell to $80.16 \%$ in the fifth year (Figure 2).

Another recurrence risk factor is non-radical excision of the primary lesion. In our study all recurrences were observed after non-radical excision (tumour cells visible in the line of excision). There were no recurrences after excision with a narrow margin. For non-radical excisions, the recurrence-free rate plateaued at 90,08\% after one year and fell to $86.33 \%$ after 5 years (Figure 3 ). In our study we did not find a connection between the margin of non-radical excision (lateral or deep) and the recurrence rate $(p=0.924)$. There were no statistically significant differences between localization of BCC $(p=0.731)$ tumour staging ( $p=0.229)$ and local recurrence. In the analysed group of patients, there was no statistically significant correlation between the stage of advancement of the lesion and the risk of non-radical excision.

Furthermore, in our study, we found that the aggressive subtype of BCCs is the risk factor for incomplete excision $(p=0.004)$. The odds ratio for nonaggressive subtypes is 0.2. This means the risk of incomplete excision is reduced by $80 \%$, and the aggressive subtypes raise the risk five times as presented in Table 2 .

\section{Discussion}

The aim of periocular BCC surgery is to remove the tumour while maintaining eyelid functionality and facial aesthetics. There are several guidelines concerning minimal excision margins of periocular BCC. The UK National Multidisciplinary Guidelines recommend that noninfiltrative $\mathrm{BCC}<2 \mathrm{~cm}$ should be excised with a margin of $4-5 \mathrm{~mm}$. Smaller margins $(2-3 \mathrm{~mm})$ may be taken in case of limited reconstructive options [4]. Moreover, the American Academy of Dermatology and NCCN both recommend excision of periocular BCC with a $4 \mathrm{~mm}$ margin $[3,5]$. However, there are studies that show that the histopathological safety margin (HSM) can be less than $4 \mathrm{~mm}$. Chadha et al. claimed that a recurrence rate of BCC excised with $2 \mathrm{~mm}$ HSM correlates well with published results following conventional excision of BCCS [6]. Auw-Haedrich et al. in their study claimed that the excision of eyelid BCC with a $0.2 \mathrm{~mm}$ margin can prevent recurrence of periocular BCC [7].

Although MMS is recommended for high-risk BCC by NCCN and other authors, it can be too expensive and time-consuming and not every hospital can afford it. Moreover, it is not advised to use MMS for BCC with deep orbital invasion as it is difficult to obtain correctly oriented specimens from orbital soft tissues [8]. Because of the limited access to MMS, surgical excision of BCC with intraoperative confirmation of margin clearance can be successfully used.

In the analysed group of patients, the male-female ratio was $1: 1$ with a slight predominance of men and the average age was 68 years. This does not differ from the analysed literature [4, 8-10]. In our study group, BCC of the medial canthal region accounted for $55.7 \%$, followed by $32.3 \%$ for the lower eyelid. However, in the analysed literature the lower eyelid is the most common site (60$70 \%)$, followed by the medial canthal region (26-30\%) [11-13]. According to analysed literature, frequent involvement of the lower eyelid might be caused by light reflection by the cornea onto the lower lid and chronic chemical or physical tear irritation. Protection given by the eyebrow is said to reduce the risk chance of developing upper eyelid BCC $[14,15]$.

In our study, we present a group of 158 patients with periocular BCC that underwent standard surgical excision with a margin of $4 \mathrm{~mm}$ of surrounding healthy tissue as the safety margin. The recurrence rate after complete excision of BCC ranges from 5\% to 14\% [15]. In the analysed literature, the recurrence rate after incomplete excision of primary periocular BCC ranges from $12 \%$ to $18.9 \%[9,11,16]$. In our study, the recurrence rate after incomplete excision of primary BCC is $14.3 \%$. Consequently, with this rate, according to NCCN guidelines, reoperation is needed and the treatment of choice is MMS, although margin-controlled excision of recurrent BCC can also be used [14]. In our practice, we use intraoperative histopathological examination to determine if surgical margins are clear.

We did not notice recurrences after a radical excision and excision with a narrow margin. According to AuwHaedrich et al., Chadha et al. and our studies, there is no need to reoperate patients treated for BCC with small histopathological margins [6, 7]. Nevertheless, patients should be advised to have regular check-up visits in an outpatient clinic.

The literature describes the relationship between the BCC subtype and prognosis as well as the recurrence risk after non-radical excision [10]. Based on our analysed group of patients, we found that the aggressive subtype significantly influences the risk of non-radical excision of the lesion, increasing the risk five times. According to other authors researched, recurrence after excision of nodular and superficial BCC with positive margins can vary from 3\% to $7 \%$, and for infiltrative BCC, it can reach $26 \%[15,17]$. The most common type of BCC in our study is the nodular type, and the most common type in recurrent lesions is the morpheaform type. Those data are consistent with other studies $[10,11]$.

In our study we did not find any correlation between the site of non-radical excision and the recurrence risk ( $p=0.924)$, although Miszczyk et al. found an association of a positive lateral margin with a higher risk of 
recurrence [18]. However, other authors found a correlation between the size of tumour and the recurrence risk. Recurrence in larger tumours $>2 \mathrm{~cm}$ reaches $23 \%$ to $40 \%$ and for smaller tumours decreases to $10 \%$ to $12 \%$ [15]. There are not many publications that show a correlation between the margin of non-radical excision and the recurrence rate. However, Lara et al. found a slight correlation between involvement of the deep margin and a higher recurrence rate of BCC [19]. In another study, Al Wohaib et al. proved that incomplete excision is reported more often in lateral margin causing a higher risk of recurrence [15].

Standard follow-up of the patients after BCC excision should be performed for the first 2 years every 6 months, for $3-5$ years, once a year. However, according to NCCN, recommended follow-up should be developed every 6-12 months for first 5 years, and then at least annually for life [3].

\section{Conclusions}

There is no need to reoperate patients with a narrow margin of BCC excision. However, these patients should be advised to conduct regular self-checks with respect to new lesions in other localizations. Aggressive BCC subtypes significantly affect the risk of a non-radical excision. Marking the subtype of BCC during the incisional biopsy is very important since it helps to choose the best method of treatment. Aggressive subtypes of BCC should result in more frequent patient check-ups in an outpatient clinic during the first years after surgery. Observations of the patient should be extended over time because of the possibility of recurrence even up to 5 years.

\section{Conflict of interest}

The authors declare no conflict of interest.

\section{References}

1. Abbas OL, Borman H. Basal cell carcinoma: a single-center experience. ISRN Dermatol. 2012; 2012: 246542.

2. Hamada S, Kersey T, Thaller VT. Eyelid basal cell carcinoma: non-Mohs excision, repair, and outcome. Br J Ophthalmol 2005; 89: 992-4.

3. NCCN Clinical Practice Guidelines in Oncology Head and Neck Cancer, version 1. 2019, January 29, 2019.

4. Newlands C, Currie R, Memon A, et al. Non-melanoma skin cancer: United Kingdom National Multidisciplinary Guidelines. J Laryngol Otol 2016; 130: S125-32.

5. Bichakjian CK, John YS. Guidelines of care for the management of basal cell carcinoma. J Am Acad Dermatol 2018; 78: 560-78.

6. Chadha V, Wright M. Small margin excision of periocular basal cell carcinomas. Br J Ophthalmol 2009; 93: 803-6.

7. Auw-Haedrich C, Frick S, Boehringer D, et al. Histologic safety margin in basal cell carcinoma of the eyelid. Correlation with recurrence rate. Ophthalmology 2009; 116: 802-6.
8. Shi Y, Jia R, Fan X. Ocular basal cell carcinoma: a brief literature review of clinical diagnosis and treatment. OncoTargets Therapy 2017; 10: 2483-9.

9. Collin JR. Basal cell carcinoma in the eyelid region. $\mathrm{Br} J \mathrm{Oph}$ thalmol 1976; 60: 806-9.

10. Ho SF, Brown L, Bamford M, et al. 5 years review of periocular basal cell carcinoma and proposed follow-up protocol. Eye 2013; 27: 78-83.

11. Ben Simon GJ, Lukovetsky S, Lavinsky F, et al. Histological and clinical features of primary and recurrent periocular basal cell carcinoma. ISRN Ophthalmology 2012; 2012: 354829.

12. Lindgren G, Diffey BL, Larkö O. Basal cell carcinoma of the eyelids and solar ultraviolet radiation exposure. $\mathrm{Br} J \mathrm{Oph}$ thalmol 1998; 82: 1412-5.

13. Totir M, Alexandrescu C, Pirvulescu R, et al. Clinical, histopathological and therapeutical analysis of inferior eyelid basal cell carcinomas. J Med Life 2014; 7 Spec No. 4 (Spec Iss 4): 18-22.

14. Luz FB, Ferron C, Cardoso GP. Surgical treatment of basal cell carcinoma: an algorithm based on the literature. An Bras Dermatol 2015; 90: 377-83.

15. Al Wohaib M, Al Ahmadi R, Al Essa D, et al. Characteristics and factors related to eyelid basal cell carcinoma in Saudi Arabia. Middle East Afr J Ophthalmol 2018; 25: 96-102.

16. Pieh S, Kuchar A, Novak P, et al. Long term results after surgical basal cell carcinoma excision in the eyelid region. Br J Ophthalmol 1999; 83: 85-8.

17. Iljin A, Zieliński T, Antoszewski B, et al. Clinicopathological analysis of recurrent basal cell carcinoma of the eyelid. Adv Dermatol Allergol 2016; 33: 42-6.

18. Miszczyk J, Charytonowicz J, Dębski T, et al. Incomplete excision of basal cell carcinoma (BCC) in the head and neck region: to wait, or not to wait? Adv Dermatol Allergol 2017; 34: 607-11.

19. Lara F, Santamaría JR, Garbers LEF de M. Recurrence rate of basal cell carcinoma with positive histopathological margins and related risk factors. An Bras Dermatol 2017; 92: 58-62. 